\title{
EL USO TIC'S EN EL PROCESO DE ENSEÑANZA-APRENDIZAJE DEL ALEMÁN COMO LENGUA EXTRANJERA
} EN LA UNIVERSIDAD

\section{THE USE OF ICTS IN THE} TEACHING-LEARNING PROCESS OF GERMAN AS A FOREIGN LANGUAGE AT THE UNIVERSITY

\section{Dayson David Ahumada Ebratt ${ }^{1}$}

Universidad del Norte

\section{RESUMEN}

El presente artículo de reflexión tuvo como objetivo describir el uso de las TICS en la enseñanza del alemán como lengua extranjera en la educación superior. Para ello el estudio se enfocó en una revisión documental cuyos autores hicieron énfasis en el uso de las TICS para fortalecer las competencias comunicativas de los estudiantes, como Cariaga (2018), Chao (2014), Hernández (2017), entre otros. En este orden, la investigación, inscrita en el paradigma cualitativo, tuvo entre sus resultados que

las TIC'S permiten tanto al docente como al estudiante, tener acceso a diversos materiales y niveles de interacción, ya sea docenteestudiante o estudiantes - estudiantes, lo que sin duda fortalece el proceso de enseñanza aprendizaje. Asimismo, durante la revisión de la literatura se pudo constatar que el estudio de las herramientas TIC'S a nivel universitario amerita una investigación mucho más detallada y rigurosa, puesto que es un tema con una gran cantidad de matices.

\section{PALABRAS CLAVE:}

lenguas extranjeras, alemán, educación, TIC'S, aprendizaje. 
* Este artículo se desprende de la tesis de maestría titulada "El uso TIC'S en el proceso de enseñanza-aprendizaje del alemán como lengua extranjera en la universidad del Norte de Barranquilla, Colombia". Presentada en la UNEATLANTICO-UNINI-PR.

\section{ABSTRACT}

This reflective article aimed to describe the use of ICTs in the teaching of German as a foreign language in higher education. For this purpose, this study focused on a documentary review whose authors emphasized the use of ICTs to strengthen students' communicative competences, such as Cariaga (2018), Chao (2014), Hernández (2017), among others. In this order, this documentary research, inscribed in the qualitative paradigm had among its results that ICT'S allow both the teacher and the student, to have access to various materials and levels of interaction, either teacher-student or students - students, which undoubtedly strengthens the teaching - learning process. Likewise, during the literature review, it was found that the study of ICT tools at the university level deserves a much more detailed and rigorous research, since it is a topic with a large number of nuances.

\section{KEY WORDS:}

foreign languages, German, education, ICT's, learning.

\section{SUMÁRIO}

Este artigo reflexivo teve como objetivo descrever o uso das TICs no ensino do alemão como língua estrangeira no ensino superior. Para tanto, este estudo focalizou uma revisão documental cujos autores enfatizaram o uso das TICs para fortalecer as competências comunicativas dos estudantes, como Cariaga (2018), Chao (2014), Hernández (2017), entre outros. Nesta ordem, esta pesquisa documental, inscrita no paradigma qualitativo teve entre seus resultados que as TIC'S permitem tanto ao professor quanto ao aluno, ter acesso a vários materiais e níveis de interação, seja professor-aluno ou alunos alunos, o que sem dúvida fortalece o processo de ensino - aprendizagem. Também, durante a revisão da literatura, descobriu-se que o estudo das ferramentas TIC a nível universitário, merece uma pesquisa muito mais detalhada e rigorosa, pois é um tema com muitas nuances.

\section{PALAVRAS-CHAVE:}

línguas estrangeiras, alemão, educação, TIC, aprendizagem.

\section{INTRODUCCIÓN}

A lo largo de la historia del ser humano hay momentos en los que el progreso y desarrollo de las ciencias ha marcado pautas en la sociedad. Existe una relación entre progreso y la ciencia en tanto la segunda impulsa y acelera al primero. Las ciencias a su vez han sido creadoras y propulsoras de todas las tecnologías que se emplean hoy en día. En este sentido, la tecnología se ha implementado en distintas áreas como la bancaria, la judicial, entre otras, con el fin de realizar, agilizar y facilitar procesos y operaciones que hace algunos años solo eran posibles visitando las entidades físicas. Ahora bien, en el ámbito educativo la incursión de la tecnología tiene un impacto que va más allá de hacer operaciones mecánicas porque se busca emplear tales herramientas con propósitos de enseñanza-aprendizaje más complejos.

Con relación al uso de las TICS en el ámbito de la enseñanza - aprendizaje, Burbat (2016) explica:

Actualmente, la situación en el sector educativo se caracteriza por un incremento constante de las TIC, lo que también se refleja en el contexto de la enseñanza-aprendizaje de LE. Así, pues, se nos ofrece un gran número y una amplia variedad de las herramientas digitales que mayoritariamente se proporcionan o bien a través de Internet o bien mediante software 
específico. La oferta es tan enorme que da la sensación de que es prácticamente imposible, estar a la última, también debido al progreso tan acelerado en el ámbito de la informática. Pero no solamente hay una multitud de herramientas digitales con las que se pueden perseguir también un sinfín de diferentes objetivos. (p. 38)

Es evidente que en el fragmento se resaltan los enormes alcances que pueden tener las tecnologías de la información en el ámbito de la enseñanza de lenguas extranjeras y que representan un recurso valiosísimo tanto para el docente como para el estudiante. De igual forma, la inclusión de las TICS en la enseñanza de L2, es enfocada por Cassany citado en Lima y Araújo (2018), quien explica:

También cabe aclarar que introducir tecnología no equivale necesariamente a innovar en metodología. Son dos cosas diferentes. Cambiar una clase presencial magistral por unos vídeos de clases expositivas colgados en una plataforma digital puede ser digitalización, pero no supone ningún avance metodológico. Igualmente, organizar una clase con un juego de rol o un debate cara a cara, tomando nota con papel y lápiz, puede no ser muy tecnológico, pero es una práctica activa centrada en el alumno -y muy innovadora en muchos casos (p. 1281)

Es decir, que el incluir las TICS en el aula de L2 es una tarea compleja, que no obliga al docente a replantear toda su planeación sino a realizarle ajustes con ayuda de estos recursos tecnológicos no perdiendo de vista que la meta es lograr un proceso de enseñanza-aprendizaje enriquecedor tanto para el estudiante como para el mismo docente. Las TICS en la enseñanza de L2 es un tema sumamente complejo que llama a los académicos para que hagan una revisión exhaustiva y contextualizándola desde el aula de clases.

Debido a todo esto, en el presente trabajo se tiene como objetivo analizar uso de las TIC
(Tecnologías de información y comunicación) en el proceso de enseñanza-aprendizaje del alemán como lengua extranjera en una universidad ubicada en Barranquilla, Colombia. $Y$ es que el uso de las TICS en la enseñanza de lenguas extranjeras ha sido un tema que ha despertado el interés de diversos investigadores como Rodríguez (2016), quien ha resaltado los distintos beneficios que han sido reportados por los docentes que emplean las tecnologías en sus praxis:

Además, algunas investigaciones han demostrado su influencia positiva en la motivación del alumno por el aprendizaje, más aún, según el "Barómetro de idiomas 2011" realizado por el Instituto de Empresa sobre los hábitos de aprendizaje de segundas lenguas, el $37 \%$ de los alumnos opina que Internet es uno de los mejores recursos para aprender un idioma. Este informe concluye que la inmensa mayoría de los estudiantes conoce su uso (el Messenger, los foros, youtube o el correo electrónico suelen ser de uso habitual) lo que "contribuye a crear en el aula una dinámica enormemente positiva" (p. 571)

Es palpable, que la autora muestra como el uso de las tecnologías incentivan el aprendizaje en los estudiantes, porque los mismos pupilos están habituados a usar las tecnologías de la información y comunicación, como por ejemplo el internet y las distintas plataformas. Es un hecho ampliamente conocido, que si el estudiante tiene conocimientos previos su proceso de aprendizaje será mucho más fácil y rápido, eso es precisamente lo que la autora quiere destacar, el uso de las tecnologías de la comunicación no es un tema ni ajeno ni desconocido para los estudiantes, por eso las convierte en unas excelentes aliadas en el aprendizaje - enseñanza de lenguas extranjeras como el alemán, por ejemplo. 


\section{TECNOLOGÍAS DE LA INFORMACIÓN Y COMUNICACIÓN}

Los programas educativos fundamentados en las tecnologías de información y comunicación han desarrollado una cultura académica a nivel mundial en donde la persona encuentra la posibilidad de cualificar su profesión a través de capacitaciones y estudios de una forma tranquila y efectiva. Es aquí donde se vuelve imperativo el uso de estas tecnologías, convirtiéndose en el eje fundamental de dichos programas y dando un giro a los métodos de educación convencional, en donde la modalidad presencial había sido tradicionalmente un factor indispensable (Brown, 2014; Izquierdo Et. All, 2017; Torres y Yépez, 2018; Pastran, Gil y Cervantes, 2020)

Para determinar cuáles son las ventajas del uso de las TICS es necesario primero hacer exposición detallada de sus definiciones asumiendo posturas para direccionar esta investigación. Muchos autores han desarrollado conceptos en torno a estas tecnologías, sin embargo, los preceptos teóricos en este ámbito son dinámicos y se actualizan con el paso del tiempo.

Las TICS se definen según Pérez et al (2018) ${ }^{2}$ como un espacio de socialización donde los jóvenes se desplazan para comunicarse con otros, obtener información y conocimiento, construir su propia identidad y sentirse parte de un colectivo; mientras que para Erazo $(2009)^{3}$ y Burbat (2016), se han convertido en eje estructural pues permite nuevos modos de percepción de la información y el lenguaje, conllevando a una trasformación cultural globalizada en donde ya no solo tienen efecto para consultar y compartir información sino para generar nuevos conocimientos.

Entre las ventajas que se registran de las TICs resalta que los usuarios tengan capacidades inmediatas para acceder a información, compararla, intercambiarla, presentarla y transmitir datos efectivamente.

Lo anterior es coherente con lo que afirma Cobo (2009):

Dispositivos tecnológicos (hardware y software) que permiten editar, producir, almacenar, intercambiar y transmitir datos entre diferentes sistemas de información que cuentan con protocolos comunes. Estas aplicaciones, que integran medios de informática, telecomunicaciones y redes, posibilitan tanto la comunicación y colaboración interpersonal (persona a persona) como la multidireccional (uno a muchos o muchos a muchos). Estas herramientas desempeñan un papel sustantivo en la generación, intercambio, difusión, gestión y acceso al conocimiento (p. 312)

De acuerdo con esta postura, se podría decir que los medios tecnológicos actuales no solo contribuyen a una mejor presentación de los conocimientos, sino que los usuarios desarrollarían formas mejoradas de percibir, analizar e interiorizar los contenidos.

Por su parte, Montenegro (2016) expone con respecto al uso de las TICS en la educación:

para satisfacer las exigencias de la sociedad actual se deben crear mecanismos donde el estudiante se motive por aprender, tratar de involucrarlo en un ambiente de aprendizaje dinámico, colaborativo, significativo y autónomo. Para crear estos ambientes educativos se requiere que el docente se apropie de las tecnologías de información y comunicación TIC, como un recurso didáctico primordial en su proceso de enseñanza y a través de ellos, adquiera múltiples competencias, con el objetivo de mejorar los resultados de aprendizaje en los estudiantes. (p. 16) 
Es evidente que para la autora, las TICS contribuyen al crecimiento y mejoramiento de los procesos de enseñanza - aprendizaje dentro y fuera del aula clases. Asimismo, destaca los variados alcances que pueden tener las tecnologías en cuanto a la promoción del aprendizaje autónomo e independiente de los estudiantes.

Por otra parte, se les denomina así a los programas e instrumentos que organizan, comunican y divulgan el conocimiento a través de medios especializados, los cuales a través del tiempo han facilitado la interactividad de los usuarios quienes dejan de ser pasivos transformándose en protagonistas de la llamada "sociedad de la información" tomando gran relevancia en las actividades académicas y científicas actuales, dicho esto, el autor de esta investigación concuerda con en asegurar que estas tecnologías permiten la transmisión de información en cualquier momento y a cualquier lugar, esta competencia coloca a esta nueva cultura digital sobre muchos métodos antiguos de compartir los conocimientos entre docentes u aprendices (Orozco, 2013; Vega, 2018; Cacheiro, 2018)

Por su parte, Cassany citado por Lima y Araújo (2018)

Los docentes de ELE pueden: usar Facebook para crear un grupo cerrado con los alumnos, para intercambiar sus producciones escritas $u$ orales; o pueden crear un grupo en WhatsApp, para incrementar la exposición a la lengua meta. También pueden desarrollar una dinámica vinculada con un tablón común de fotos en Pinterest o en Instagram. Se pueden desarrollar una comunidad de blogueros, en la que cada alumno, o cada grupo de alumnos, postean periódicamente sus textos -animados por el docente - y hacen comentarios a los blogs de los alumnos. Algunos docentes vinculan diversos recursos (diccionarios, traductores, plataformas de escritura) a un espacio propio, para que los alumnos tengan un acceso más directo a estos recursos curados por un experto. Las posibilidades son muy diversas y lo bueno es que permite adaptarlas a cada contexto y grupo de alumnos. (p. 1277)

Es evidente, que para el autor la enseñanza de lenguas extranjeras puede beneficiarse muchísimo del uso de las herramientas TICS y sus distintos recursos como las aplicaciones y las redes sociales. Se observa, que destaca el rol mediador del docente y la capacidad de promover la autonomía e independencia de los estudiantes en sus propios procesos aprendizaje. El acceso a redes sociales, por ejemplo, le permite tanto al estudiante como al profesor acceder a un sinfín de materiales que sin duda enriquecen el proceso de adquisición de L2.

\section{LAS TICS SON MÁS QUE SOLO HERRAMIENTAS}

Como ya se ha expuesto, las TICS ofrecen grandes ventajas en el proceso de aprendizaje de los estudiantes y en el de enseñanza para los docentes. En este sentido, para autores como Conejo y Herrara citados por Garrido (2015) explican que las TICS permiten a estudiantes que no puedan trasladarse al extranjero, vivir experiencias comunicativas reales de aprendizaje de la lengua en contexto, al afirmar que:

Aquellos alumnos que no puedan o no quieran viajar a un país extranjero, las nuevas tecnologías les dan acceso a espacios de interacción, información y materiales auténticos que de otra manera no estarían a su alcance. EI MCER establece el "saber aprender" como una de las características individuales del aprendiente de lenguas. En este sentido, las TIC son una excelente aportación por su potencial para fomentar el aprendizaje autónomo del alumno (p. 9) 
Es observable, que en el fragmento se explica cómo las TICS no solo ayudan al aprendizaje de la lengua extranjera, sino que además fomenta la autonomía en el aprendiz, característica y ventaja del uso de las tecnologías que ha sido ampliamente reportada por distintos autores que más adelante se mencionaran.

Asimismo, la llamada autonomía que promueven las tecnologías de la información y comunicación ha sido tema de interés de estudiosos como Burbat (2016) quien expone:

De esta manera, la enseñanza y el papel del profesor en general recobran valor. Enseñar se concibe a través de la intervención y el control del proceso de aprendizaje por parte del profesor según las circunstancias que se les presenten. Sus funciones de asesorar, orientar y servir de modelo se consideran necesarias hasta que los estudiantes sean capaces de desprenderse. En definitiva, a nuestro juicio, en el marco de la enseñanza-aprendizaje el concepto de autonomía del enfoque psicológico-evolutivo no sólo constituye el estado o la aptitud final, sino además el objetivo de aprendizaje (p. 42)

Para esta autora, la autonomía que predican las TICS debe tener inicialmente por lo menos, un acompañamiento del docente de lenguas porque si bien es cierto que las tecnologías ponen a disposición del educando un sinfín de información sin la orientación pertinente del docente, es muy probable que el estudiante no aproveche del todo el potencial de tal información. Igualmente, la autonomía es un concepto que constantemente se reinventa en el ámbito educativo debido a las múltiples herramientas y estrategias que día a día de diseñan pensando en la mejora del proceso de aprendizaje.

Igualmente, la autora Burbat (2016) destaca que las TICS no tienen la capacidad humana de detectar detalles o percibir las emociones que muestran los estudiantes durante su proceso de aprendizaje porque a menudo estas plataformas digitales tienen muy poco margen de error en las actividades que les muestran a sus usuarios, es decir que son actividades sumamente estructuradas y cerradas relacionadas con aspectos gramaticales o de léxico, esto se afirma partiendo del siguiente fragmento:

Debido a que la tecnología informática no es capaz de diagnosticar los errores de forma detallada, tampoco proporciona un apoyo cognitivo significativo al usuario. Asimismo, dispone solo de un margen estrecho de errores, una dificultad que además se multiplica si consideramos el enorme potencial de errores en el plano semántico y pragmático de LE. Por este motivo, abundan los ejercicios interactivos enfocados al aspecto estructural de la lengua que a menudo se critican por su apego a las prácticas behavioristas (p. 47)

Es necesario resaltar que el presente estudio surge de la curiosidad investigativa del presente autor con respecto a las posibles ventajas y beneficios que tiene el uso de las TIC'S en la enseñanza y aprendizaje de lenguas extranjeras, específicamente en el alemán, en espacios de educación superior.

\section{LAS TICS EN LA ENSEÑANZA DE LENGUAS EXTRANJERAS COMO EL ALEMÁN}

Según el Instituto Cervantes (2014) en su informe, determinó que aproximadamente un $(1,1 \%)$ de la población mundial habla alemán, ocupando así el 4to puesto de las lenguas más habladas del mundo distintas al inglés. $Y$ es que a lo largo de Europa se habla el idioma Alemán, ya sea debido a su legado literario que van de la mano de sus autores emblemáticos como Goethe o de los más recientes como Süskind; además de los incontables músicos, filósofos, pintores, entre otros, que indudablemente hacen de esta lengua un referente obligatorio para todo aquel que desee tener una perspectiva clara y completa de la cultura europea y es que, 
para entender la complejidad de una cultura es necesario conocer la lengua en la que fue creada (Bueno, 2016)

Sin duda alguna, el idioma alemán tiene una importancia no solo en Europa sino a nivel mundial, debido a que, en términos de las ciencias, por ejemplo, la mayoría de los cimientos científicos de innumerables disciplinas siguen estando en alemán, sin dejar de mencionar que, aun en la actualidad gran parte del progreso científico en distintas tecnologías sigue desarrollándose en Alemania obviamente en lengua alemana. Es por esto que, se afirma que el hablar este idioma germano no solo puede tener un impacto positivo en lo laboral puesto que Alemania es la mayor potencia económica dentro de la Unión Europea con muchas sedes globales aumentando las posibilidades laborales de quienes dominan esta lengua, sino que también, ofrece grandes oportunidades a los estudiantes debido a que este país otorga becas a través de diferentes entidades para diversos fines académicos como el DAAD, ERASMUS y KAAD entre otras, todo esto se afirma partiendo de los postulados de la Casa Cultural Colombo Alemana (2019).

Todas estas razones, han despertado el interés de muchísimas personas a nivel mundial por aprender el idioma alemán y los colombianos no se han mantenido indiferentes ante esta tendencia. Según el Ministerio de Educación de Colombia (2018) en el informe disponible en su portal online, existe una clara tendencia por parte de los institutos educativos, de ofrecer cursos y preparación en lenguas extranjeras, especialmente en la lengua alemana. Incluyéndola como segundo o tercer idioma dentro de plantilla curricular académica o incluso como curso libre para acentuar sus posibilidades de sobresalir en el ámbito laboral actual cada vez más y más competitivo.

Esta tendencia está en concordancia con la dinámica del mundo de hoy en día, que está en constante cambio debido a las distintas transformaciones que ha traído la globalización, cambios donde no solamente cobra importancia el aprendizaje de los idiomas, sino también el uso de la tecnología como una herramienta que favorece el aprendizaje de una lengua en gran medida por la forma en que se dinamiza el aprendizaje - enseñanza, proceso que ya no debe abarcar solo el espacio de un aula sino que puede transcender gracias a las distintas alternativas que ofrece la web y los recursos de las TIC'S como tal (Flórez, et. Al. 2017)

En este sentido, las TIC'S no se pueden excluir en el aprendizaje de un idioma, esto evidentemente, puede representar una tarea compleja de realizar, puesto que se requiere del aprendizaje de diferentes destrezas no solo por parte de los estudiantes sino de los docentes también; dichas habilidades, podrían no solo mejorar la interacción docente-estudiante, también lo haría con la de estudiante-estudiante.

Igualmente, Rodríguez (2016) expone con respecto a estas herramientas que, serían excelentes recursos para agilizar el aprendizaje de competencias comunicativas como la oralidad en la LE, cuando afirma que "Resultan efectivas para facilitar y propiciar el desarrollo de las destrezas orales a la hora de aprender un idioma extranjero" (p. 2). Además de este aspecto, el factor motivación también se refuerza con el uso de las TIC'S. Asimismo Rodríguez (2016) plantea "Los alumnos se muestran muy positivos ante el uso de las TIC. Apuntan que las actividades con herramientas Web son más motivadoras." (p.10). En otras palabras, el uso de las tecnologías en la enseñanza de lengua extranjera, trae beneficios en lo académico y también, en lo afectivo.

Sin embargo, aún con este panorama tan positivo, no todos los profesores son expertos o hacen uso de tales herramientas, otros docentes incluso se muestran muy reacios, al uso de la tecnología durante sus sesiones de 
alemán debido a que, consideran que estas prácticas y herramientas tecnológicas no van en consonancia con sus valores de la praxis docente. En este sentido, Cassany citado por Lima y Araújo (2018) expone:

Laeducación no deja de ser una disciplina en parte basada en la tradición, en lo que la experiencia y la historia han mostrado que es bueno -o que los docentes creemos que es bueno--. Entonces, muchos docentes viven adheridos al pasado o a sus conocimientos desarrollados en la época del papel, cuando estudiaron y se formaron como profesionales. Todos conocemos algunos tecnofóbicos que están en contra de las tecnologías y que defienden el uso del papel, de manera más explícita o velada. $Y$ frente a ellos también encontramos docentes más jóvenes, más abiertos, con una actitud tecnofílica, que están encantados de introducir tecnología en sus clases y de avanzar hacia la digitalización. (p. 1281)

Ahora bien, actualmente los estudiantes están expuestos a mucha información y diferentes recursos tecnológicos los cuales deberían ser utilizados en pro del proceso de enseñanzaaprendizaje. Es por esto que, se hace necesario disponer de una estrategia que les permita a los estudiantes de lenguas extranjeras como el alemán, emplear la tecnología para ayudarles a solventar las debilidades que presenten en su aprendizaje, lo que evidentemente hace pertinente la realización de este trabajo de investigación.

\section{CONCLUSIONES}

Para autores como Cassany como fue citado por Lima y Araújo (2018) las TICS pueden transformarse en las mejores aliadas del docente dentro y fuera del aula, pero es necesario que los profesores reciban una mejor orientación en cuanto a las bondades de las TICS, al respecto expone:
En general, creo que los docentes no hemos recibido suficiente formación para utilizar con eficacia las tecnologías en el aula. El cambio es muy lento y requiere digestión. Varios estudios sugieren que los docentes necesitamos unos 3 años para cambiar de paradigma, para hacernos totalmente digitales. (p. 1281)

Y es que en la revisión de la literatura que se realizó en esta investigación, se observó que existen muy pocos trabajos relacionados con el acompañamiento que han recibido los docentes de lenguas extranjeras en el desarrollo y manejo de las TICS. Lo cual resulta sumamente curioso si se compara con la cantidad de trabajos que estudian las ventajas y desventajas del uso de las TICS en el aula.

Es decir, se centra mucho la investigación en la práctica, pero poco en el adiestramiento que recibió o no el docente para emplear las TICS en su praxis. Salvo por Borromeo (2016) quien explora el uso de las redes sociales y cómo su uso no es tan atractivo para algunos docentes, la mayoría de los estudios son enfocados en los estudiantes.

Por su parte, Cordeiro como fue citada en Lima y Araújo (2018) explica que la necesidad del uso de las TICS radica en el efecto motivador que tiene sobre los estudiantes y que indudablemente le facilita al docente su proceso de enseñanza, al postular lo siguiente:

Creo que la incorporación de las redes sociales en el aula y en el aprendizaje está muy relacionada a la necesidad de ofrecer a los estudiantes oportunidades de comunicación auténticas, que conectan con sus intereses reales en lengua extranjera. Las redes sociales representan un espacio de práctica real de la lengua, sea ella materna o extranjera, ya que conecta a personas de todo el mundo en una escala jamás vista, que puede tener implicaciones positivas en el proceso de aprendizaje de una LE. Entre ellas, 
destacaría su uso como un material motivador (p. 1281)

De igual forma, otros autores han explorado el efecto motivacional que tienen las TICS en los estudiantes como los ya mencionados en este trabajo como Angulo y Guatibonza, 2008; Pérez y Pastran, 2019; Brescó y Verdú , 2014; Cala, Díaz, Espí y Tituaña, 2018 y así se podrían mencionar muchísimos más estudiosos que se han enfocado en el desglose de las ventajas y desventajas que trae para el proceso de aprendizaje tanto de pupilos como de docentes.

$Y$ es que el tema de las TICS no es tan nuevo como se cree, sus diversas aplicaciones en la vida diaria y en el ámbito educativo ha demandado otra forma de ver no solo a la educación sino a la gama de procesos cognitivos que están involucrados en el aprendizaje y en la enseñanza.

Destaca especialmente el carácter motivacional que está implícito en la utilización de las TICS. Al respecto, Borromeo (2016) explica:

A pesar de todo lo mencionado anteriormente, se mantienen una discreta línea entre el uso y el no uso de las redes sociales con fines académicos, sean redes especializadas o de uso general. Entre los factores que mantienen a las redes sociales en un umbral de no inclusión están: la no integración al plan de estudios o al currículo, la resistencia al cambio de paradigma, el desconocimiento, la falta de destreza tecnológica necesaria para explotar sus posibilidades y el limitado acceso para ciertos grupos de personas (p. 45)

Es entonces necesario que los docentes reciban un mayor acompañamiento en cuanto al manejo de herramientas TICS como las redes sociales, debido a que los estudiantes ya manejan con mucha destreza este tipo de aplicaciones en su vida cotidiana lo que sin duda es un gran aliciente para incluirlos en los procesos de aprendizaje.

\section{REFERENCIAS BIBLIOGRÁFICAS}

Angulo, A., y Guatibonza, A. (2008) Descripción del uso pedagógico de herramientas tecnológicas provistas por la pontificia universidad javeriana en la comunidad académica de la licenciatura en lenguas modernas en la enseñanza y aprendizaje de una lengua extranjera (inglés $y$ francés) (tesis de Pregrado). Pontificia Universidad Javeriana, Bogotá.

Brescó, E. y Verdú, N. (2014). Valoración del uso de las herramientas colaborativas wikispaces y google drive en la educación superior. Edutec-e, (49).

Borromeo, C. (2016). Redes sociales para la enseñanza de idiomas: el caso de los profesores. Revista de Medios y Educación, (48), 41-50. Recuperado de https://www.redalyc.org/articulo. oa?id=368/36843409004

Bueno, G. (2016) El mito de la cultura. Recuperado de http://www.fgbueno.es/ gbm/gb1996mc.htm

Burbat, R. (2016) El aprendizaje autónomo y las TIC en la enseñanza de una lengua extranjera: ¿Progreso o retroceso?. Porta Linguarum, 26, 3751. Recuperado de https://www.ugr. es/ portalin/articulos/PL numero26/ ART3 Ruth\%20Burbat.pdf

Brown, D. (2014). Principles of language learning and teaching. Madrid: Pearson.

Cala, R., Díaz, L., Espí, N. y Tituaña, J. (2018). El Impacto del Uso de Pizarras Digitales Interactivas (PDI) en el Proceso de Enseñanza Aprendizaje. Un Caso de Estudio en la Universidad de Otavalo. Información tecnológica, 29(5), 61-70. 
Cariaga, R. (2018). Experiencias en el uso de las TIC: Análisis de relatos de docentes. Ciencia, docencia y tecnología, (56), 131-155.

Cobo, J. (2011). El concepto de tecnologías de la información. Benchmarking sobre las definiciones de las TIC en la sociedad del conocimiento. Revista de Estudios de Comunicación, 14 (27), 295-318.

Chao, K. (2014). Estrategias didácticas mediadas con TIC en un curso de expresión oral francesa. Actualidades Investigativas en Educación, 14(2), 1 - 30.

Erazo, E. (2009). De la construcción histórica de la condición juvenil a su transformación contemporánea. Revista Latinoamericana de Ciencias Sociales, Niñez y Juventud, 7(2), 1303 -1329.

Fernández, A. y Rivero, M. (2014). Las plataformas de aprendizajes, una alternativa a tener en cuenta en el proceso de enseñanza aprendizaje. Revista Cubana de Informática Médica, 6 (2), 207-221.

Flórez, M.,Aguilar, A. y Hernández, Y. (2017) Sociedad del conocimiento, las TIC y su influencia en la educación. Recuperado de http://www.revistaespacios.com/ a17v38n35/17383539.html

Garrido, M. (2015) Las TIC y la enseñanza de lenguas extranjeras (Tesis de pregrado). Universidad de Jaén. Jaén, España.

Hernández, R. (2017). Impacto de las TIC en la educación: Retos y Perspectivas. Propósitos y Representaciones, 5 (1), 325 - 347.

Izquierdo, J., De-la-Cruz, V., Aquino, S., Sandoval, M. y García, V. (2017) La enseñanza de lenguas extranjeras y el empleo de las TIC en las escuelas secundarias públicas. Comunicar, XXV, (50), 33-41. Recuperado de http://www.redalyc.org/ articulo.oa?id=15849613003

Lima, G. y Araújo, J. (2018) El uso de las redes sociales para el desarrollo de la enseñanza de ele: el investigador y el profesor. Un diálogo entre estos dos sujetos a través de la entrevista con Daniel Cassany y Dayane Cordeiro. Trabalhos em Lingüística Aplicada, 57(2),1274-1296. Doi: https://dx.doi.org $\underline{110.1590 / 010318138651882364651}$

Montenegro,A. (2016)Apropiacióndetecnologías de información y comunicación (tic) en docentes de inglés del nivel beginner del centro de idiomas de la universidad de Nariño para los procesos de enseñanza (Tesis de maestría). Universidad Libre. Bogotá, Colombia.

Ministerio de Educación de Colombia (2018) Ruta de apropiación de las TIC en el desarrollo profesional docente. Recuperado de http://www.mineducacion.gov. co/1621/w3-article-233944.html.

Nord, J. H., Riggio, M. T., y Paliszkiewicz, J. (2017). Social and Economic Development through Information and Communications Technologies: Italy. Journal of Computer Information Systems, 57 (3), 278-285.

Pastran, M., Gil, N. y Cervantes, D. (2020). En tiempos de coronavirus: las TIC'S son una buena alternativa para la educación remota. Revista Boletín Redipe, 9(8), 158-165. https://doi.org/10.36260/rbr. v9i8.1048

Pérez, A. y Pastran, M. (2019) ¿Qué es escritura académica desde la percepción del estudiante?. Conferencia llevada a cabo en el III Congreso Internacional de 
Investigación Lingüística en Medellín, Antioquia.

Pérez, M., Marín, B. y Arias, A. (2018). Socialización de jóvenes a través de las TIC en una institución educativa de Antioquia. Pensamiento Psicológico, 16(2), 59-72. .

Rodríguez, N. (2016) ¿Las TIC como mediadoras en la enseñanza-aprendizaje de lenguas extranjeras? Opción, 32 (10). 569-588. Recuperada de https://www.redalyc.org/ articulo.oa?id=31048901031

Torres, M. y Yépez, D. (2018) Aprendizaje cooperativo y TIC y su impacto en la adquisición del idioma inglés. Revista mexicana de investigación educativa, 23(78), 861-882 